\title{
Detection of OSR2, VAV3, and PPFIA3 Methylation in the Serum of Patients with Gastric Cancer
}

\author{
Wen-han Li, ${ }^{1}$ Zhang-jian Zhou, ${ }^{1}$ Tian-he Huang, ${ }^{1}$ Kun Guo, ${ }^{2}$ Wei Chen, ${ }^{1}$ \\ Ying Wang, ${ }^{1}$ Hao Zhang, ${ }^{1}$ Yong-chun Song, ${ }^{1}$ and Dong-min Chang ${ }^{1}$ \\ ${ }^{1}$ The First Affiliated Hospital of Xi'an Jiaotong University, Xian, Shaanxi, China \\ ${ }^{2}$ No. 2 Subsidiary Hospital of No. 4 Military Medical University, Xian, Shaanxi, China
}

Correspondence should be addressed to Dong-min Chang; dr_changdm@sina.com

Received 28 December 2015; Revised 19 February 2016; Accepted 2 March 2016

Academic Editor: Stamatios Theocharis

Copyright (C) 2016 Wen-han Li et al. This is an open access article distributed under the Creative Commons Attribution License, which permits unrestricted use, distribution, and reproduction in any medium, provided the original work is properly cited.

\begin{abstract}
Aim. This study was to evaluate the diagnostic value of OSR2, VAV3, and PPFIA3 hypermethylation in gastric cancer (GC) patients. Patients and Methods. By using methylation-specific polymerase chain reaction (MSP), we detected the methylation status in tissue and serum samples from 48 gastric cancer (GC) patients and 25 normal individuals. Results. We found that OSR2, VAV3, and PPFIA3 were methylated in $70.8 \%(34 / 48), 54.2 \%(26 / 48)$, and $60.4 \%(29 / 48)$ of GC tissue, respectively. On the contrary, those genes were barely methylated in their paired paracancerous histological normal tissues (PCHNTs) (all $P$ values $<0.01$ ). We next analyzed the methylated OSR2, VAV3, and PPFIA3 in serum DNA. Compared with 25 normal individuals, those three genes were significantly hypermethylated in GC patients serum samples (all $P$ values $<0.01$ ). Regarding their diagnostic value in serum samples, the combined sensitivity of at least one positive among the three markers in serum was $83.3 \%$, with a specificity of $88 \%$. Conclusion. Our test suggested that methylation of OSR2, VAV3, and PPFIA3 genes in serum sample may offer a good alternative in a simple, promising, and noninvasive detection of GC.
\end{abstract}

\section{Introduction}

Although the incidence of gastric cancer (GC) has declined in the past decades, it is still one of the most common malignancies worldwide. According to related data, the annual diagnosis number of GC approximately reaches one million; among them $42 \%$ are from China $[1,2]$. As GC is usually asymptomatic until advanced stage, it is often associated with poor treatment outcome and low 5-year survival rate. Therefore, an ideal screening tool to detect GC with high sensitivity and specificity has a high priority. Upper endoscopy can distinguish between cancerous and noncancerous conditions by performing biopsies of suspicious areas, therefore being considered as the golden standard for GC detection. However, considering its invasive operations, high-risk patients of GC are reluctant to take this approach as a regular examination. Serum tumor markers, such as carcinoembryonic antigen (CEA), carbohydrate antigen (CA) 19-9, and carbohydrate antigen (CA) 724, have been widely applied in clinical practice. However, none of them are suitable for early detection of GC. Till now, there is no ideal diagnostic method with relatively high sensitivity that could be applied in clinical screening for GC.

As we all know, GC is a multistep process and accumulating data have elucidated that epigenetic alterations, especially DNA methylation, play an important role in GC initiation. By silencing the tumor suppressor genes which play a key role in DNA repair, cell adhesion, cell cycle control, and apoptosis [3], DNA hypermethylation at CpG islands in or near promoter regions contributes a lot during the process of carcinogenesis. Moreover, it has been shown that changes of methylation in body fluids paralleled other somatic tissues and therefore are thought to be connected with certain cancers $[4,5]$. On the basis of those studies above, many attempts have been made to investigate biomarkers in serum, urine, and sputum in various malignancies [6-11]. As for GC, many researchers thought serum DNA-based technique is a promising alternative for its relatively noninvasive operations 
TABLE 1: Methylation-specific primers of OSR2, PPFIA3, and VAV3.

\begin{tabular}{lcccc}
\hline Primer set & Forward primer & Reverse primer & $\begin{array}{c}\text { Annealing } \\
\text { temperature } \\
\left({ }^{\circ} \mathrm{C}\right)\end{array}$ & $\begin{array}{c}\text { Amp } \\
\text { size }(\mathrm{bp})\end{array}$ \\
\hline OSR2-M & CGTAGCGCGTGGGATTTTAC & CCAATACTAACAAACCGAAACG & 57 & 100 \\
OSR2-U & GGTTTAGGAGGATGAAGTGT & CACCCTATAACCACCTTTCCCACA & 58 & 85 \\
VAV3-M & GGTTTTTTTTTCGCGCGGGATC & ACGAAAAACGCGCGAAACTCG & 57 & 57 \\
VAV3-U & GGTTTTTTTTTGTGTGGGATT & CACAAAAAACACACAAAACTCA & 57 & 139 \\
PPFIA3-M & GGTATGTGGTCGTTTGTC & CGAATTACTAATACCGATCTCG & 98 \\
PPFIA3-U & GGTATGTGGTTGTTTGTT & CAAATTACTAATACCAATCTCA & 54 & 98 \\
\hline
\end{tabular}

as well as a convenient tissue to assay for constitutional methylation. Many studies have been made to explore the feasibility of the use of serum as a biomarker for certain cancers $[4,5,12-18]$.

The selection of the candidate gene for analyzing is crucial to improving the sensitivity and specificity of methylation DNA test. Current microarray technology provides us with an opportunity for high-throughput unbiased methylation analysis of a large number of CpG sites [19]. By using an Infinium HumanMethylation 450 BeadChip array, Zong and colleagues [20] identified three genes (OSR2, VAV3, and PPFIA3) that were hypermethylated in GC tissue. Oddskipped related 2 (OSR2), which contains DNA-binding $\mathrm{C} 2 \mathrm{H} 2$-type zinc finger domains in the C-terminal half, plays an important role in cellular quiescence and proliferation under epigenetic regulation [21, 22]. Additionally, recent articles have reported that VAV3 (a member of VAV gene family which plays an important role in the process of tumor development and metastasis) and PTPRF-Interacting Protein Alpha-3 (PPFIA3) may associated with the tumorigenesis and development of GC $[20,23]$. In the present study, we sought to explore the feasibility of DNA methylation status of OSR2, VAV3, and PPFIA3 as a noninvasive screening tool for GC.

\section{Materials and Methods}

2.1. Collection of Tissue and Peripheral Blood Samples. In order to reduce bias, we designed this experiment as a blinded assay. All sample collection and preservation were taken care of by a person who did not participate in the follow-up studies. Patients with primary gastric cancer who participated in this study were recruited consecutively from February 2012 to August 2013. The study material consisted of 48 tumor tissue samples, paired paracancerous histological normal tissues (PCHNTs) which are obtained during curative surgery, and the patients' whole blood samples preoperatively. In the meantime, another 25 blood samples from healthy individuals were also obtained. None of the experimental subjects had received prior gastric resection or preoperative chemotherapy/radiation therapy. All samples were immediately frozen and stored at $-80^{\circ} \mathrm{C}$ until DNA was extracted. In order to reduce bias, samples were randomly coded before processing. All patients voluntarily joined this study with written informed consents to have their biologic specimens analyzed. This study was announced by the Ethical Committee of the First Affiliated Hospital of Xi'an Jiaotong University.

2.2. DNA Isolation. DNA was extracted from tissues $(10 \pm$ $1 \mathrm{mg}$ ) with the TIANamp Genomic DNA Kit and for serum samples $(400 \mu \mathrm{L})$ by use of TIANamp Blood DNA Kit (Tiangen, China). All procedures were strictly carried out according to the manufacturer's instructions. The concentration of DNA was measured by ultraviolet spectrophotography and the quality of DNA was tested by PCR amplification of the human $\beta$-actin.

2.3. Bisulfite Modification. As to bisulfite genomic DNA modification, $2000 \mathrm{Ng}$ of DNA was modified by EpiTect Bisulfite Kit (Qiagen) to convert all unmethylated cytosine to uracil. The bisulfite-treated DNA was eluted in $20 \mu \mathrm{L}$ of TE buffer and stored at $-20^{\circ} \mathrm{C}$ until being processed.

2.4. Methylation-Specific Polymerase Chain Reaction (MSP). After the bisulfite treatment, we used methylation-specific PCR to testify the methylation status of the OSR2, PPFIA3, and VAV3 promoter. The primers specific to methylated and unmethylated sequences and annealing temperature are summarized in Table 1 . We repeated each experiment at least three times in order to reduce false results.

Water without DNA was used as a negative control. Product was visualized by electrophoresis in a $2 \%$ agarose gel and analyzed by a gel imaging system. The methylation pattern result was judged by the distribution of visible bands.

2.5. CEA, CA19-9, and CA-724 Measurements. Normal levels of CEA, CA19-9, and CA-724 were defined as $<3.4 \mathrm{ng} / \mathrm{mL}$, $<39 \mathrm{U} / \mathrm{mL}$, and $<9.8 \mathrm{U} / \mathrm{mL}$, respectively. The tests were done independently at the clinical laboratory in the First Affiliated Hospital of Xi'an Jiaotong University College of Medicine.

2.6. Statistical Analysis. The methylation status of those three genes and all other qualitative variables were expressed as frequencies and percentages (\%). The relationship between the methylation status of serum samples and the clinicopathological characteristics was calculated using Fisher's 


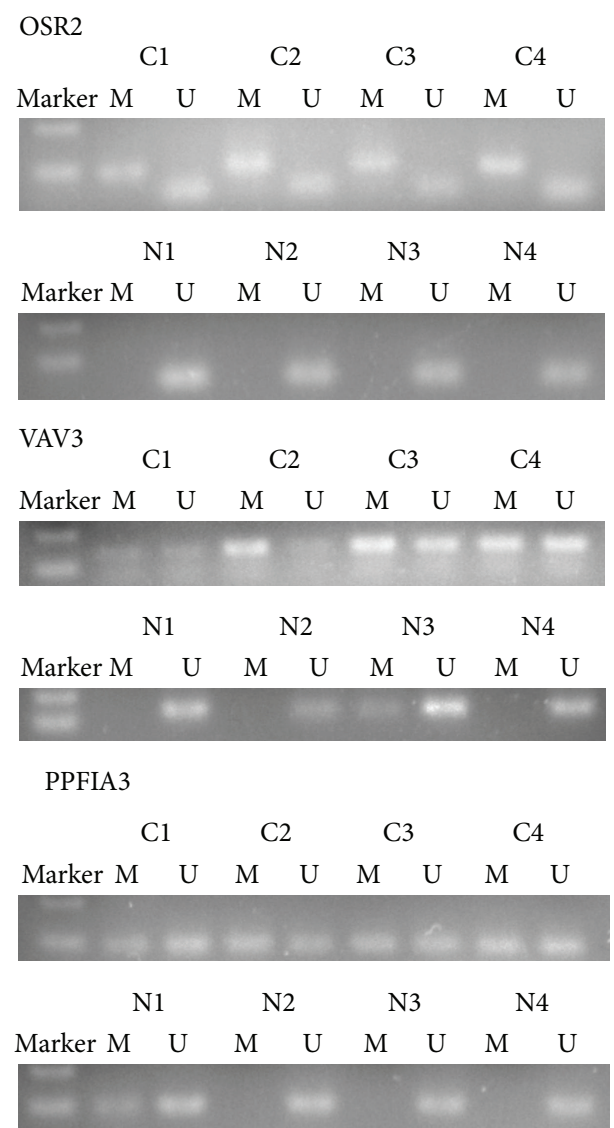

FIGURE 1: Detection of methylated (M) and unmethylated (U) OSR2, VAV3, and PPFIA3 in tissue of gastric cancer $(\mathrm{Cl}-\mathrm{C} 4)$ and paired paracancerous histological normal mucosa (N1-N4).

exact test or chi-square test. The correlation of each gene methylation status between GC and their matched gastric nontumorous tissues or serum samples was calculated using Fisher's exact test or chi-square test. Statistical analyses were performed with the SPSS 13.0 software. $P$ values $<0.05$ (twosided) were considered statistically significant.

\section{Results}

3.1. Patient Characteristics. In order to explore the methylation status of OSR2, VAV3, and PPFIA3 in GC, 48 GC patients (including 39 males and 9 females) and 25 healthy individuals (including 18 males and 7 females) were enrolled in our study. The mean age of GC patients and healthy controls was $56.75 \pm$ 10.6 and $53.48 \pm 14.43$, respectively. There was no significant difference with respect to age and gender between cases and controls (age: $P=0.55$; gender: $P=0.365$ ).

3.2. Gene Promoter Hypermethylation in Tissue Samples. We first examined the methylation status of OSR2, VAV3, and PPFIA3 in the tissue DNA of GC patients and PCHNTs. The results of representative MSP cases are shown in Figure 1. All of the three genes detected by MSP in GC group showed positive results in unmethylated promoter regions, indicating
TABLE 2: The frequency of promoter hypermethylation of OSR2, VAV3, and PPFIA3 in tissue and serum samples.

\begin{tabular}{lcccc}
\hline & \multicolumn{2}{c}{ Tissue samples } & \multicolumn{2}{c}{ Serum samples } \\
& GC & PCHNTs & GC & Normal \\
\hline OSR2 & $70.8 \%(34 / 48)$ & $4 \%(1 / 25)$ & $62.5 \%(30 / 48)$ & $8 \%(2 / 25)$ \\
VAV3 & $54.2 \%(26 / 48)$ & $0 \%(0 / 25)$ & $45.8 \%(22 / 48)$ & $0 \%(0 / 25)$ \\
PPFIA3 & $60.4 \%(29 / 48)$ & $4 \%(1 / 25)$ & $56.3 \%(27 / 48)$ & $4 \%(1 / 25)$ \\
\hline
\end{tabular}

PCHNTs: paired paracancerous histological normal tissues.

that there exist some nonneoplastic cells in cancer tissue samples. The prevalence of methylation of these 3 genes was shown in Table 2. Out of $48 \mathrm{GC}$ tissue samples, $34(70.8 \%)$, $26(54.2 \%)$, and $29(60.4 \%)$ exhibited OSR2, VAV3, and PPFIA3 hypermethylation, respectively. On the contrary, in the PCHNTs group, hypermethylation of those three genes was rarely found (OSR2: 4\%; VAV3: 0\%; PPFIA3: 4\%). The data indicated that methylated OSR2, VAV3, and PPFIA3 DNA in tissue were significantly higher in GC patients than those of controls (all $P$ values $<0.01$ ).

3.3. Gene Promoter Hypermethylation in Serum Samples. To further investigate whether those three genes' methylation could be used as a biomarker for GC, we detected the methylation frequency of those three genes in serum samples between GC patients and healthy controls. Methylation of OSR2, VAV3, and PPFIA3 was detected in $30(62.5 \%), 22$ (45.8\%), and $27(56.3 \%)$ of the serum of 48 GC patients, respectively (Table 2; Figure 2), whereas those genes were weakly methylated in the healthy control group (OSR2: 8\%; VAV3: 0\%; PPFIA3: 4\%). By analyzing the data in tissue samples, we found that all serum samples with aberrant methylation were accompanied with methylation in the corresponding tumor samples. The above evidence suggested that OSR2, VAV3, and PPFIA3 genes are highly methylated in the serum of GC patients compared to healthy ones (all $P$ values $<0.01)$. Altogether, more than $83.3 \%(40 / 48)$ of patients have hypermethylation in at least one of the three analyzed markers in their serum DNA, in contrast to 3 of $25(12 \%)$ healthy controls $(P<0.01)$ (Table 3$)$. The results show that the comethylation reaches $83.3 \%$ sensitivity and $88 \%$ specificity and therefore may be considered as a panel for the early detection of GC.

3.4. Association of Methylation Status in Serum with Clinicopathological Parameters. A summary of the methylation status of the three genes in serum samples and the clinicopathological parameters is shown in Table 4. No significant correlation was found of overall methylation with patients' demographic data, including age, gender, pathological differentiation, depth of tumor invasion, and lymph node metastasis. However, patients with advanced stage disease exhibited a higher serum methylation frequency in VAV3 gene $(P=0.019)$. Interestingly, the methylated OSR2, VAV3, and PPFIA3 in serum exist at the early stage of GC (TNM stages I and II) with a relatively high percentage (OSR2:59.1\%; VAV3: $36.4 \%$; PPFIA3: $45.5 \%$ ) and therefore could be used to screen GC. 


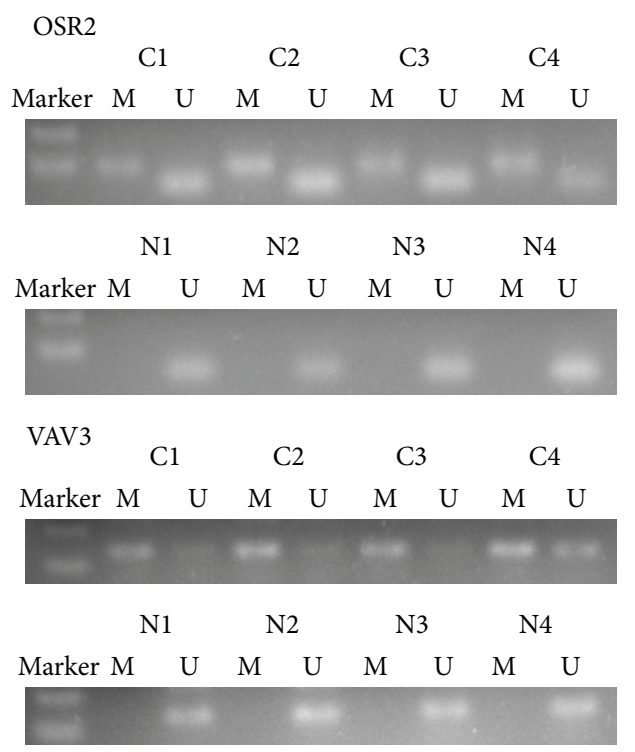

PPFIA3

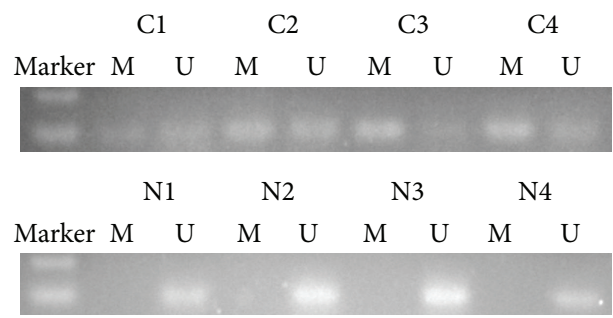

Figure 2: Detection of methylated (M) and unmethylated (U) OSR2, VAV3, and PPFIA3 in serum of gastric cancer (C1-C4) and normal individuals (N1-N4).

TABLE 3: The positive rate of at least one hypermethylated gene in serum samples.

\begin{tabular}{lcccc}
\hline \multicolumn{3}{c}{ OSR2 + VAV3 + PPFIA3 methylation } & Positive percent & $P$ value \\
& Positive & Negative & & \\
\hline GC & 40 & 8 & $83.3 \%$ & $<0.01^{*}$ \\
Normal & 3 & 22 & $12 \%$ & \\
\hline
\end{tabular}

When the markers were used in combination, the test was considered to be positive if one marker reached the threshold and negative if all three markers were negative.

Using chi-square for this statistic.

${ }^{*}$ Statistically significant.

3.5. Relationship between Serum Tumor Markers and Serum Methylation. We subsequently compared the diagnostic value between gene methylation and serum tumor markers (Table 5). The sensitivity of combined detection of CEA, CA19-9, and CA-724 reached 33.3\%, which was significantly lower than that of MSP assay (33.3\% versus $83.3 \%, P<$ 0.01 ). Moreover, when we stratified the TNM stages and analyzed the diagnostic value between those two methods in each stage, we found that detection of OSR2, VAV3, and PPFIA3 hypermethylation showed significantly higher sensitivity than that of serum tumor markers (Table 5). Thus, detection of those three genes' methylation in serum was indicated to be more sensitive compared to combined detection of CEA, CA19-9, and CA-724 in the early stage of GC.

\section{Discussion}

In recent years, epigenetic alterations, especially DNA methylation, seem hot to researchers. Studies have shown that aberrant epigenetic modifications occur at the early stage of cancer initiation and play an important role in human carcinogenesis. Therefore, specific methylation biomarkers hold the promise to act as useful tools for early cancer detection. Moreover, those changes could also be detected in the nontarget tissue, especially in blood samples [24, 25]. This gives us a feasibility to detect cancer in a relatively less invasive way.

There have been several studies that previously reported that genes were hypermethylated in GC tissue but were hypomethylated or unmethylated in healthy gastric mucosae [26-30]. However, the majority of studies focused on single gene, which may lead to a relatively low sensitivity in detecting GC cancer. In this study, we evaluated the methylation status of three genes together in both tissue and serum samples to improve the sensitivity. According to Zong et al. [20] research, by using an Infinium HumanMethylation 450 BeadChip array, they identified that OSR2, VAV3, and PPFIA3 are barely methylated in normal cells but highly methylated in GC cells. To further assess the clinical value of methylated OSR2, VAV3, and PPFIA3, we first analyzed those genes' methylation status in GC tissue samples or PCHNTs samples. For each gene (OSR2, VAV3, and PPFIA3), aberrant methylation was significantly more frequent in GC tissue compared to PCHNTs ( $70.8 \%$ versus $4 \%, 54.2 \%$ versus $0 \%$, and $60.4 \%$ versus $4 \%$, resp.). We next analyzed serum samples from 48 GC individuals and 25 healthy controls. The result shows that aberrant methylation of those three genes was significantly more frequent in the serum of GC patients compared to healthy subjects (all $P$ values $<0.05$ ). The combined sensitivity of at least one positive among the three markers in serum samples reached satisfactory outcome with $83.3 \%$ in tumor serum samples (40/48). The analysis between methylation status in the serum and clinicopathological data demonstrated that the methylation status of VAV3 was significantly more frequent in the serum DNA of patients with advanced cancer (TNM stages III and IV) than those with early-stage cancer (TNM stages I and II) $(P=0.019)$. This phenomenon may be caused by the limited sample size, or the percentage of tumor DNA is usually higher in the sera of these advanced GC patients. Finally, our study compared the diagnosis value between promoter hypermethylation and serum tumor markers that are currently used in clinical practice. The result showed that the comethylation of those three genes had a significantly higher sensitivity and specificity than CEA, CA19-9, and CA724 (83.3\% versus 33.3\%) (Table 5). Further studies using a greater number of samples needed to be performed to elucidate the diagnostic power of those markers in serum.

For any ideal diagnostic approaches, candidate biomarkers and methods should be with high sensitivity and specificity and relatively noninvasive and could be applied in 
TABLE 4: Correlation between OSR2, VAV3, and PPFIA3 hypermethylation status in serum of GC patients and clinicopathological parameters.

\begin{tabular}{|c|c|c|c|c|c|c|c|}
\hline \multirow{2}{*}{ Parameters } & \multirow{2}{*}{ Number of cases } & \multicolumn{2}{|c|}{ OSR2 } & \multicolumn{2}{|c|}{ VAV3 } & \multicolumn{2}{|c|}{ PPFIA3 } \\
\hline & & Methylation & $P$ value & Methylation & $P$ value & Methylation & $P$ value \\
\hline \multicolumn{8}{|l|}{ Age } \\
\hline$<60$ & 26 & $16(61.5 \%)$ & \multirow{2}{*}{$0.881^{\mathrm{a}}$} & $13(50.0 \%)$ & \multirow{2}{*}{$0.529^{\mathrm{a}}$} & $14(53.8 \%)$ & \multirow{2}{*}{$0.715^{\mathrm{a}}$} \\
\hline$\geq 60$ & 22 & $14(63.6 \%)$ & & $9(40.9 \%)$ & & $13(59.1 \%)$ & \\
\hline \multicolumn{8}{|l|}{ Gender } \\
\hline Male & 39 & $23(59.0 \%)$ & \multirow{2}{*}{$0.504^{\mathrm{a}}$} & $19(48.7 \%)$ & \multirow{2}{*}{$0.186^{\mathrm{a}}$} & $23(59.0 \%)$ & \multirow{2}{*}{$0.675^{\mathrm{a}}$} \\
\hline Female & 9 & $7(77.8 \%)$ & & $3(33.3 \%)$ & & $4(44.4 \%)$ & \\
\hline \multicolumn{8}{|l|}{ Pathological differentiation } \\
\hline Well + moderate & 19 & $13(68.4 \%)$ & \multirow{2}{*}{$0.493^{\mathrm{a}}$} & $7(36.8 \%)$ & \multirow{2}{*}{$0.583^{\mathrm{a}}$} & $11(57.9 \%)$ & \multirow{2}{*}{$0.853^{\mathrm{a}}$} \\
\hline Poor + undifferentiation & 29 & $17(58.6 \%)$ & & $13(44.8 \%)$ & & $16(55.2 \%)$ & \\
\hline \multicolumn{8}{|l|}{ Depth of tumor invasion } \\
\hline Tis, Tla, Tlb & 5 & $2(40.0 \%)$ & \multirow{4}{*}{$0.663^{\mathrm{a}}$} & $2(40.0 \%)$ & \multirow{4}{*}{$0.645^{\mathrm{a}}$} & $2(40.0 \%)$ & \multirow{4}{*}{$0.694^{\mathrm{a}}$} \\
\hline $\mathrm{T} 2$ & 7 & $5(71.4 \%)$ & & $2(28.6 \%)$ & & $3(42.9 \%)$ & \\
\hline T3 & 12 & $7(58.3 \%)$ & & $7(58.3 \%)$ & & $7(58.3 \%)$ & \\
\hline $\mathrm{T} 4 \mathrm{a}, \mathrm{T} 4 \mathrm{~b}$ & 24 & $16(66.7 \%)$ & & $11(45.8 \%)$ & & $15(62.4 \%)$ & \\
\hline \multicolumn{8}{|l|}{ Lymph node metastasis } \\
\hline N0 & 16 & $9(56.3 \%)$ & \multirow{4}{*}{$0.492^{\mathrm{a}}$} & $5(31.3 \%)$ & \multirow{4}{*}{$0.430^{\mathrm{a}}$} & $8(50.0 \%)$ & \multirow{4}{*}{$0.514^{\mathrm{a}}$} \\
\hline N1 & 15 & $8(53.3 \%)$ & & $7(46.7 \%)$ & & $7(46.7 \%)$ & \\
\hline N2 & 6 & $5(83.3 \%)$ & & $4(66.7 \%)$ & & $4(66.7 \%)$ & \\
\hline N3a, N3b & 11 & $8(72.7 \%)$ & & $6(54.5 \%)$ & & $8(72.7 \%)$ & \\
\hline \multicolumn{8}{|l|}{ TNM stages } \\
\hline I and II & 22 & $13(59.1 \%)$ & \multirow{2}{*}{$0.881^{\mathrm{a}}$} & $8(36.4 \%)$ & \multirow{2}{*}{$0.019^{*}$} & $10(45.5 \%)$ & \multirow{2}{*}{$0.165^{\mathrm{a}}$} \\
\hline III and IV & 26 & $17(65.4 \%)$ & & $14(53.8 \%)$ & & $17(65.4 \%)$ & \\
\hline
\end{tabular}

${ }^{a}$ Using chi-square for this statistic.

* Statistically significant.

TABLE 5: Sensitivity of serum markers for gastric cancer according to TNM stage.

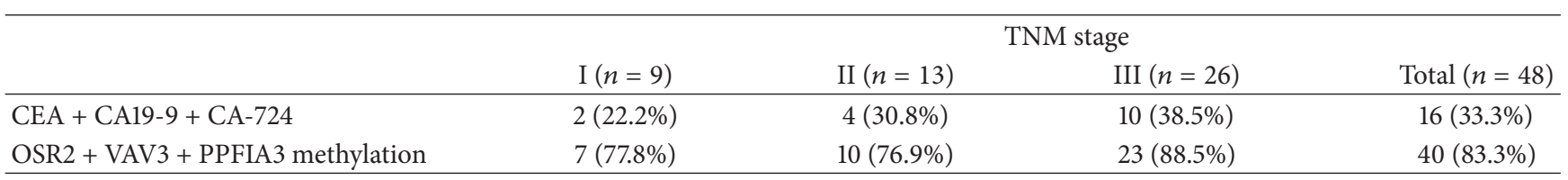

a cost-effective way. Many researchers use pyrosequencing method to detect DNA methylation to improve accuracy. However, pyrophosphate sequencing could not be widely used in clinical practice for the cost is relatively high and can be a time-consuming operation. In this study, we used conventional methylation-specific PCR (MSP) to detect DNA methylation. MSP has sufficient sensitivity to detect abnormal methylation in a large background of normal DNA [31, 32]. Besides, we detect multiple genes together to improve the sensitivity.

Although epigenetics develops fast in recent years and researchers find that DNA hypermethylation contributes a lot in GC initiation, some obstacles are still needed to be overcome before DNA methylation-based biomarkers can be adopted in clinical practice. The most important one is that guidelines, including procedures, experimental conditions, and instructions, should be standardized to improve the reproducibility of results. Even though many genes have been reported as a biomarker for the early detection, disease monitoring, prognosis, and risk assessment of cancer patients, a lot of valid experiments are still needed to be done before they truly be clinically transformative [33-35].

\section{Conclusions}

In summary, by comparing the comethylation status of OSR2, VAV3, and PPFIA3 in tissue and serum samples between GC patients and healthy individuals, we found that those three genes have a relatively high sensitivity and specificity and therefore may be used as a biomarker for noninvasive screening of GC.

\section{Competing Interests}

The authors declare that they have no competing interests. 


\section{Acknowledgments}

The authors would like to thank all participants for their helpful contribution to the present study. This work was supported in part by a grant from the Scientific and Technologic Bureau of Shaanxi (2011K12-79).

\section{References}

[1] C. A. González, N. Sala, and T. Rokkas, "Gastric cancer: epidemiologic aspects," Helicobacter, vol. 18, supplement 1, pp. 34-38, 2013.

[2] H. Zhang, L.-L. Sun, Y.-L. Meng et al., "Survival trends in gastric cancer patients of Northeast China," World Journal of Gastroenterology, vol. 17, no. 27, pp. 3257-3262, 2011.

[3] D. S. Shames, J. D. Minna, and A. F. Gazdar, "DNA methylation in health, disease, and cancer," Current Molecular Medicine, vol. 7, no. 1, pp. 85-102, 2007.

[4] J.-P. J. Issa, N. Ahuja, M. Toyota, M. P. Bronner, and T. A. Brentnall, "Accelerated age-related CpG island methylation in ulcerative colitis," Cancer Research, vol. 61, no. 9, pp. 3573-3577, 2001.

[5] T. Maekita, K. Nakazawa, M. Mihara et al., "High levels of aberrant DNA methylation in Helicobacter pylori-infected gastric mucosae and its possible association with gastric cancer risk," Clinical Cancer Research, vol.12, no. 3 I, pp. 989-995, 2006.

[6] J.-Y. Choi, S. R. James, P. A. Link et al., "Association between global DNA hypomethylation in leukocytes and risk of breast cancer," Carcinogenesis, vol. 30, no. 11, pp. 1889-1897, 2009.

[7] X.-P. Zou, B. Zhang, X.-Q. Zhang, M. Chen, J. Cao, and W.J. Liu, "Promoter hypermethylation of multiple genes in early gastric adenocarcinoma and precancerous lesions," Human Pathology, vol. 40, no. 11, pp. 1534-1542, 2009.

[8] K. B. Sriram, V. Relan, B. E. Clarke et al., "Diagnostic molecular biomarkers for malignant pleural effusions," Future Oncology, vol. 7, no. 6, pp. 737-752, 2011.

[9] T. Wu, E. Giovannucci, J. Welge, P. Mallick, W.-Y. Tang, and S.M. Ho, "Measurement of GSTP1 promoter methylation in body fluids may complement PSA screening: a meta-analysis," British Journal of Cancer, vol. 105, no. 1, pp. 65-73, 2011.

[10] L. Li, J.-Y. Choi, K.-M. Lee et al., "DNA methylation in peripheral blood: a potential biomarker for cancer molecular epidemiology," Journal of Epidemiology, vol. 22, no. 5, pp. 384394, 2012.

[11] J. Zhou, Y.-H. Shi, and J. Fan, "Circulating cell-free nucleic acids: promising biomarkers of hepatocellular carcinoma," Seminars in Oncology, vol. 39, no. 4, pp. 440-448, 2012.

[12] K. Hibi, T. Goto, A. Shirahata et al., "Detection of TFPI2 methylation in the serum of gastric cancer patients," Anticancer Research, vol. 31, no. 11, pp. 3835-3838, 2011.

[13] L. Chen, L. Su, J. Li et al., "Hypermethylated FAM5C and MYLK in serum as diagnosis and pre-warning markers for gastric cancer," Disease Markers, vol. 32, no. 3, pp. 195-202, 2012.

[14] A. Shirahata, K. Sakuraba, Y. Kitamura et al., "Detection of vimentin methylation in the serum of patients with gastric cancer," Anticancer Research, vol. 32, no. 3, pp. 791-794, 2012.

[15] M. Barchitta, A. Quattrocchi, A. Maugeri, M. Vinciguerra, and A. Agodi, "LINE-1 hypomethylation in blood and tissue samples as an epigenetic marker for cancer risk: a systematic review and meta-analysis," PLoS ONE, vol. 9, no. 10, Article ID 0109478, 2014.
[16] Y.-C. Wu, P. Lv, J. Han et al., "Enhanced serum methylated p16 DNAs is associated with the progression of gastric cancer," International Journal of Clinical and Experimental Pathology, vol. 7, no. 4, pp. 1553-1562, 2014.

[17] Y. Zhang, H. J. Su, K. F. Pan et al., "Methylation status of blood leukocyte DNA and risk of gastric cancer in a high-risk Chinese population," Cancer Epidemiology Biomarkers \& Prevention, vol. 23, no. 10, pp. 2019-2026, 2014.

[18] I. Balgkouranidou, D. Matthaios, A. Karayiannakis et al., "Prognostic role of APC and RASSF1A promoter methylation status in cell free circulating DNA of operable gastric cancer patients," Mutation Research/Fundamental and Molecular Mechanisms of Mutagenesis, vol. 778, pp. 46-51, 2015.

[19] M. Bibikova, Z. Lin, L. Zhou et al., "High-throughput DNA methylation profiling using universal bead arrays," Genome Research, vol. 16, no. 3, pp. 383-393, 2006.

[20] L. Zong, N. Hattori, Y. Yoda et al., "Establishment of a DNA methylation marker to evaluate cancer cell fraction in gastric cancer," Gastric Cancer, 2015.

[21] S. Kawai and A. Amano, "Odd-skipped related 2 is epigenetically regulated in cellular quiescence," Biochemical and Biophysical Research Communications, vol. 396, no. 4, pp. 831836, 2010.

[22] S. Kawai, Y. Abiko, and A. Amano, "Odd-skipped related 2 regulates genes related to proliferation and development," Biochemical and Biophysical Research Communications, vol. 398, no. 2, pp. 184-190, 2010.

[23] B.-B. Tan, M.-M. Zhang, Y. Li et al., "Inhibition of Vav3 gene canpromote apoptosis of human gastric cancer cell line MGC803 by regulating ERK pathway," Tumor Biology, 2015.

[24] S. E. Cottrell and P. W. Laird, "Sensitive detection of DNA methylation," Annals of the New York Academy of Sciences, vol. 983, pp. 120-130, 2003.

[25] V. V. Vlassov, P. P. Laktionov, and E. Y. Rykova, "Circulating nucleic acids as a potential source for cancer biomarkers," Current Molecular Medicine, vol. 10, no. 2, pp. 142-165, 2010.

[26] G. H. Kang, S. Lee, J.-S. Kim, and H.-Y. Jung, "Profile of aberrant $\mathrm{CpG}$ island methylation along the multistep pathway of gastric carcinogenesis," Laboratory Investigation, vol. 83, no. 5, pp. 635641, 2003.

[27] M. F. G. de Maat, C. J. H. van de Velde, N. Umetani et al., "Epigenetic silencing of cyclooxygenase-2 affects clinical outcome in gastric cancer," Journal of Clinical Oncology, vol. 25, no. 31, pp. 4887-4894, 2007.

[28] M. Ye, B. Xia, Q. Guo, F. Zhou, and X. Zhang, "Association of diminished expression of RASSF1A with promoter methylation in primary gastric cancer from patients of central China," $B M C$ Cancer, vol. 7, article 120, 2007.

[29] P. Gu, X. Xing, M. Tänzer et al., "Frequent loss of TIMP-3 expression in progression of esophageal and gastric adenocarcinomas," Neoplasia, vol. 10, no. 6, pp. 563-572, 2008.

[30] K. Hosoya, S. Yamashita, T. Ando, T. Nakajima, F. Itoh, and T. Ushijima, "Adenomatous polyposis coli $1 \mathrm{~A}$ is likely to be methylated as a passenger in human gastric carcinogenesis," Cancer Letters, vol. 285, no. 2, pp. 182-189, 2009.

[31] J. G. Herman, J. R. Graff, S. Myöhänen, B. D. Nelkin, and S. B. Baylin, "Methylation-specific PCR: a novel PCR assay for methylation status of CpG islands," Proceedings of the National Academy of Sciences of the United States of America, vol. 93, no. 18, pp. 9821-9826, 1996. 
[32] B. Vogelstein and K. W. Kinzler, "Digital PCR," Proceedings of the National Academy of Sciences of the United States of America, vol. 96, no. 16, pp. 9236-9241, 1999.

[33] C. M. Sturgeon, B. R. Hoffman, D. W. Chan et al., "National academy of clinical biochemistry laboratory medicine practice guidelines for use of tumor markers in clinical practice: quality requirements," Clinical Chemistry, vol. 54, no. 8, pp. el-e10, 2008.

[34] S. U. Meyer, M. W. Pfaffl, and S. E. Ulbrich, "Normalization strategies for microRNA profiling experiments: a 'normal' way to a hidden layer of complexity?" Biotechnology Letters, vol. 32, no. 12, pp. 1777-1788, 2010.

[35] Y. Toiyama, Y. Okugawa, and A. Goel, "DNA methylation and microRNA biomarkers for noninvasive detection of gastric and colorectal cancer," Biochemical and Biophysical Research Communications, vol. 455, no. 1-2, pp. 43-57, 2014. 


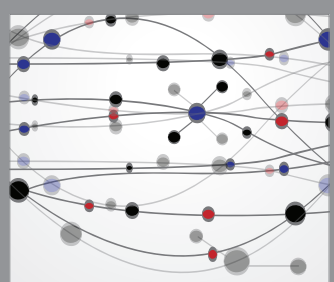

The Scientific World Journal
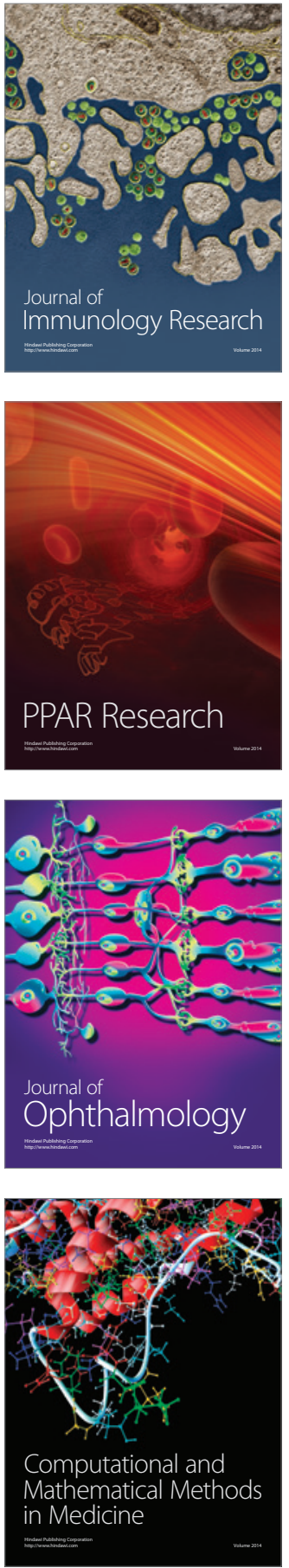

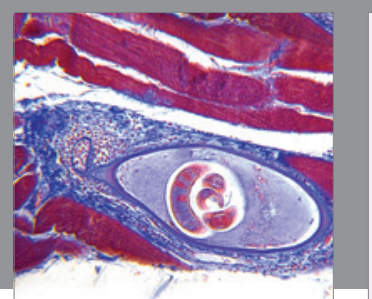

Gastroenterology Research and Practice

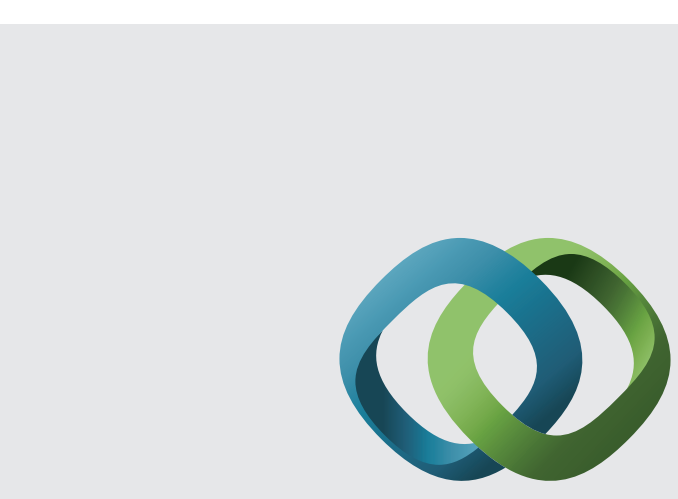

\section{Hindawi}

Submit your manuscripts at

http://www.hindawi.com
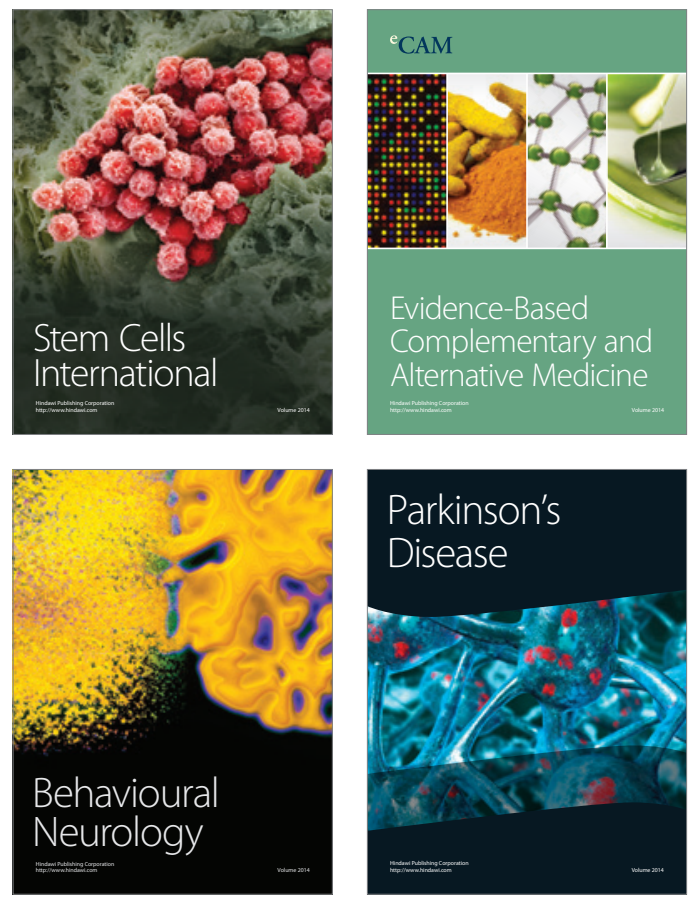
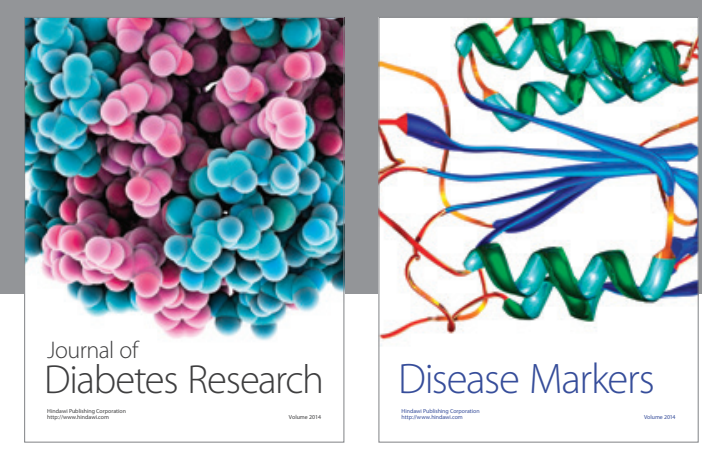

Disease Markers
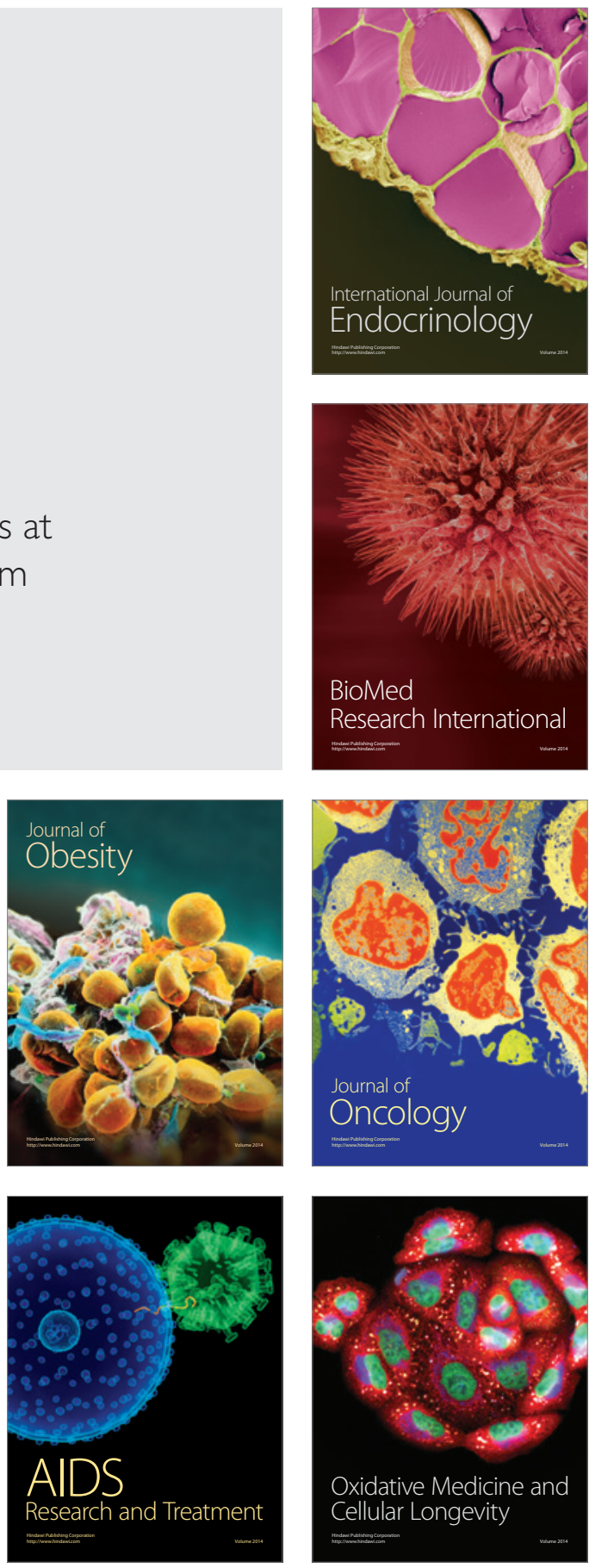